TRANSCUTANEOUS

ELECTRICAL ACUPOINT STIMULATION IMPROVES THE OUTCOMES OF IN VITRO FERTILIZATION: A PROSPECTIVE, RANDOMIZED AND CONTROLLED STUDY

Fan Qu, Fang-Fang Wang, Yan Wu, Jue Zhou, Nicola Robinson, Paul J. Hardiman, Jie-Xue Pan, Yi-Jing He, Yu-Hang Zhu, Han-Zhi Wang, XiaoQun Ye, Ke-Lin He, Long Cui, Hong-Li Zhao, Ying-Hui Ye

PII: $\quad$ S1550-8307(17)30238-0

DOI: $\quad$ http://dx.doi.org/10.1016/j.explore.2017.06.004

Reference: JSCH2214

To appear in: Explore: The Journal of Science and Healing

Cite this article as: Fan Qu, Fang-Fang Wang, Yan Wu, Jue Zhou, Nicola Robinson, Paul J. Hardiman, Jie-Xue Pan, Yi-Jing He, Yu-Hang Zhu, Han-Zhi Wang, Xiao-Qun Ye, Ke-Lin He, Long Cui, Hong-Li Zhao and Ying-Hui Ye, TRANSCUTANEOUS ELECTRICAL ACUPOINT STIMULATION IMPROVES THE OUTCOMES OF IN VITRO FERTILIZATION: A PROSPECTIVE, RANDOMIZED AND CONTROLLED STUDY, Explore: The Journal of Science and Healing, http://dx.doi.org/10.1016/j.explore.2017.06.004

This is a PDF file of an unedited manuscript that has been accepted for publication. As a service to our customers we are providing this early version of the manuscript. The manuscript will undergo copyediting, typesetting, and review of the resulting galley proof before it is published in its final citable form. Please note that during the production process errors may be discovered which could affect the content, and all legal disclaimers that apply to the journal pertain. 


\title{
Transcutaneous electrical acupoint stimulation improves the outcomes of in vitro fertilization: A prospective, randomized and controlled study.
}

\begin{abstract}
Fan $\mathrm{Qu}^{\mathrm{a}, \mathrm{b} *}$, Fang-Fang Wang ${ }^{\mathrm{a} *}$, Yan $\mathrm{Wu}^{\mathrm{a} *}$, Jue Zhou ${ }^{\mathrm{c}}$, Nicola Robinson ${ }^{\mathrm{d}}$, Paul J. Hardiman ${ }^{\mathrm{b}}$, Jie-Xue Pann ${ }^{\mathrm{e}}$, Yi-Jing He ${ }^{\mathrm{f}}$, Yu-Hang Zhu ${ }^{\mathrm{a}}$, Han-Zhi Wang ${ }^{\mathrm{a}}$, Xiao-Qun Ye ${ }^{\mathrm{a}}$, Ke-Lin He ${ }^{\mathrm{f}}$, Long Cui ${ }^{\mathrm{a}}$, Hong-Li Zhao ${ }^{\mathrm{g}}$, Ying-Hui Ye ${ }^{\mathrm{a} * *}$
\end{abstract}

${ }^{a}$ Women's Hospital, School of Medicine, Zhejiang University, Hangzhou, Zhejiang, 310006, China

${ }^{\mathrm{b}}$ Institute for Women's Health, University College London Medical School, London NW3 2PF, UK

${ }^{c}$ College of Food Science and Biotechnology, Zhejiang Gongshang University, Hangzhou, Zhejiang, 310018, China

${ }^{d}$ Faculty of Health and Social Care, London South Bank University, London, SE1 OAA, UK.

${ }^{\text {e }}$ Reproductive Medicine Center, First Affiliated Hospital of Wenzhou Medical University, Wenzhou, Zhejiang, 325000, China

${ }^{\mathrm{f}}$ Zhejiang Chinese Medical University, Hangzhou, Zhejiang, 310053, China

${ }^{\mathrm{g}}$ Hangzhou Hospital of Traditional Chinese Medicine, Hangzhou, Zhejiang, 310007, China

* The first three authors contributed equally to the present study

** Address all correspondence and requests for reprints to: $\mathrm{Dr}$ Ying-Hui Ye, Associate Professor, Women's Hospital, School of Medicine, Zhejiang University, No.1 Xueshi Road, Hangzhou, Zhejiang, 310006, China. Tel: +86 571 87061501; Fax: +86 571 87061878; E-mail: yeyh1998@hotmail.com 
Dr Fan Qu was invited to give a keynote lecture concerning the present study in the 4th Annual Meeting of the Good Practice in Traditional Chinese Medicine Research Association during 13-15 July, 2015 in Mons, Belgium, and the abstract was published as a conference abstract [European Journal of Integrative Medicine, 2015; 7 (Supplement 1): 18].

\section{Transcutaneous electrical acupoint stimulation improves the outcomes of in vitro fertilization: A prospective, randomized and controlled study.}

\section{Abstract}

Objectives: To explore whether transcutaneous electrical acupoint stimulation (TEAS) can improve the outcomes of in vitro fertilization (IVF).

Design: A prospective, randomized and controlled study.

Setting: IVF center in a university hospital.

Participants: Four hundred and eighty-one infertile patients with bilateral tubal blockage who were referred for IVF. Patients were randomized into four groups.

Intervention: TEAS was administered for $30 \mathrm{~min}$, respectively at $24 \mathrm{~h}$ before TVOR and $2 \mathrm{~h}$ before ET. The acupoints included SP10 (Xuehai, bilateral), SP8 (Diji, bilateral), LR3 (Taichong, bilateral), ST36 (Zusanli, bilateral), EX-CA1 (Zigong, bilateral), RN4 (Guanyuan), PC6 (Neiguan, bilateral) and RN12 (Zhongwan). Based on different frequencies of TEAS, patients were grouped into a TEAS-2 $\mathrm{Hz}$ group, a TEAS-100 Hz group and a TEAS-2/100 Hz group. Patients in the control group only received routine IVF treatment and no TEAS were applied on them. 


\section{ACCEPTED MANUSCRIPT}

Primary and secondary outcome measures: The number of mature oocytes, normally

fertilized oocytes and good-quality embryos were used to evaluate oocyte developmental competence of the patients. Data of clinical pregnancy rate (CPR), implantation rate (IR) and live birth rate (LBR) were also obtained. The levels of neuropeptide $Y$ (NPY), transforming growth factor alpha and granulocyte colony-stimulating factor in the follicular fluids were measured with enzyme-linked immunosorbent assay (ELISA).

Results: No significant differences were found between the control, TEAS-2 Hz, TEAS-100 Hz and TEAS-2/100 Hz groups on the numbers of metaphase II oocytes, normally fertilized zygotes, early cleavage embryos or good quality embryos $(P>0.05)$. However, the CPR, IR and LBR of the TEAS-2/100 Hz group were significantly higher than those of the other groups, respectively $(P<0.05)$. The NPY levels in the follicular fluids of TEAS-2/100 Hz group were significantly higher than those of the other groups $(P<0.05)$.

Conclusion: TEAS using a frequency of $2 / 100 \mathrm{~Hz}$ could help to improve the IVF outcomes partly by increasing NPY levels in the follicular fluids.

Key words: Transcutaneous electrical acupoint stimulation (TEAS), in vitro fertilization (IVF), clinical pregnancy rate (CPR), implantation rate (IR), live birth rate (LBR). ${ }^{1}$

\footnotetext{
TEAS: Transcutaneous electrical acupoint stimulation; IVF: In vitro fertilization; CPR: Clinical pregnancy rate; IR: Implantation rate; LBR: Live birth rate; NPY: Neuropeptide Y; ELISA: Enzyme-linked immunosorbent assay; HSG: Hysterosalpingogram; LH: Luteinizing hormone; FSH: Follicle stimulating hormone; E2: Estradiol; TT: Total testosterone; PRL: Prolactin; ART: Assisted reproductive technology; WHO: World Health Organization; CONSORT: Consolidated Standards of Reporting Trials; STRICTA: Standards for Reporting Interventions in Clinical Trials of Acupuncture; TVOR: Trans-vaginal oocyte retrieval; ET: Embryo transfer; COH: Controlled ovarian hyperstimulation; HCG: Human chorionic gonadotropin; TGF-alpha: Transforming growth factor alpha; G-CSF: Granulocyte colony-stimulating factor; GnRH: Gonadotropin-releasing hormone; CSF3:

Colony-stimulating factor 3 .
} 


\section{Introduction}

In vitro fertilization (IVF) is an important treatment for infertility, and over 300,000 cycles of IVF are performed each year in Europe. ${ }^{1}$ In recent years, although some equipment, techniques and drug therapies have been developed for improving the outcomes of IVF, the progress in developing safe and effective therapies has been limited. ${ }^{2,3}$ Many infertile women have now turned to complementary and alternative medicine as an adjuvant therapy to improve the outcomes when they undergo IVF treatment. ${ }^{4}$ Acupuncture, as an effective non-pharmacological therapy, has been chosen by many infertile couples undergoing infertility treatment. ${ }^{3,5-8}$ It is reported that "fertility problems" is the second most common health condition for which people choose acupuncture treatment in the UK. ${ }^{9}$

Acupuncture could improve the reproductive outcomes of IVF. ${ }^{10,11}$ However, in the traditional acupuncture treatment, patients' skin is pierced with the needles, which can lead to certain adverse effects and can affect acupuncture utilization. One study on the incidence of adverse effects during acupuncture therapy showed hemorrhage was the main side effect with a rate of $2.9 \% .{ }^{12}$ Another study found that bleeding $(6.1 \%)$, pain $(1.7 \%)$ and vegetative symptoms $(0.7 \%)$ were common adverse effects of acupuncture. ${ }^{13}$ These side effects may be reasons for dropout acupuncture in clinical studies ${ }^{14}$ or for decreased utilization in clinical practice.

Transcutaneous electrical acupoint stimulation (TEAS) uses self-adhesive electrodes instead of the needles to stimulate acupoints with electric current. ${ }^{15}$ Different frequencies of TEAS can generate different biological effects. Because of the advantages of noninvasiveness and painlessness, TEAS recently has been a popular treatment in many conditions, including pain, 


\section{ACCEPTED MANUSCRIPT}

depression, cancer, and withdrawal syndrome. ${ }^{16-19}$ The present prospective, randomized and controlled trial was designed to investigate whether TEAS can improve IVF outcomes for infertile women with bilateral tubal blockage and to explore the possible underlying mechanism.

\section{Methods}

\section{Subjects}

Over a five-month period (May 1, 2014 to September, 30, 2014), we screened 657 infertile women with bilateral tubal blockage who were referred to a university hospital for IVF. Among the patients screened, 162 women did not meet the inclusion criteria. Eleven women did not consent to treatment, and three women were excluded for other reasons. Finally, 481 infertile women with bilateral tubal blockage were included in the study. The subjects were randomized into a control group, a TEAS- $2 \mathrm{~Hz}$ group, a TEAS-100 $\mathrm{Hz}$ group or a TEAS-2/100 Hz group using computer generated randomization that randomized numbers into four groups with a proportion of 1:1:1:1. The random allocation sequence was concealed until the interventions were assigned. A nurse enrolled the participants and assigned them to their groups. The IVF clinicians and laboratory staff were blinded to the group assignment. Ethical permission to conduct the study was obtained from the Institutional Review Board of Reproductive Medicine. The aim and methodology of the study were explained to the patients in detail. Voluntary participation was requested and informed written consents were obtained from all of the participants. 


\section{ACCEPTED MANUSCRIPT}

The subjects included in the study were infertile women with bilateral tubal blockage who were referred to our department for IVF. The bilateral tubal blockage was determined by hysterosalpingogram (HSG). Subjects were otherwise healthy women with regular menstrual cycles and normal sex hormone levels [luteinizing hormone (LH): 3.80-20.00 IU/L; follicle stimulating hormone (FSH): 3.80-17.20 IU/L; estradiol $\left(E_{2}\right)$ : $100.00-275.00 \mathrm{pmol} / \mathrm{L}$; total testosterone (TT): $0.30-3.00 \mathrm{nmol} / \mathrm{L}$; prolactin (PRL): $5.00-30.00 \mathrm{ng} / \mathrm{mL}$ ] and no other pelvic pathology.. No structural abnormalities of uterus and ovaries were found by vaginal ultrasound or laparoscopy. None of the women had received salpingectomy or assisted reproductive technology (ART) therapy before. We excluded patients with neurologic, mood or psychiatric disorders; patients who were taking any tranquilizers; patients who were receiving acupressure or acupuncture therapy; patients with a history of smoking or drinking; and patients who underwent preimplantation genetic diagnosis or preimplantation genetic screening. All the partners of the women had normal spermiograms and sperm morphology according to the World Health Organization (WHO) criteria.

The present study adheres to the Consolidated Standards of Reporting Trials (CONSORT) statement, ${ }^{20}$ and Standards for Reporting Interventions in Clinical Trials of Acupuncture (STRICTA). ${ }^{21,22}$ The TEAS treatment was performed by two professional acupuncturists. The TEAS protocol was developed based on the clinical experience in our hospital, the literature and consultation with six experts in Chinese medicine. The TEAS devices (HANS-100B) used in the study were provided at a regular price without any direct involvement of the manufacturer (Jisheng Medical Technology Co., Ltd, Nanjing, China).

\section{Group and administration}




\section{ACCEPTED MANUSCRIPT}

Thirty-minute TEAS were administered at 24 hours before trans-vaginal oocyte retrieval

(TVOR) and 2 hours before embryo transfer (ET) respectively. Acupoints of SP10 (Xuehai, bilateral), SP8 (Diji, bilateral), LR3 (Taichong, bilateral), and ST36 (Zusanli, bilateral) were chosen before TVOR, and acupoints of EX-CA1 (Zigong, bilateral), RN4 (Guanyuan), PC6 (Neiguan, bilateral) and RN12 (Zhongwan) were chosen before ET. TEAS was applied through self-adhesive surface electrodes. The standard electrodes affiliated to the TEAS device were applied to the denuded skin and the intensity was set strong enough to elicit visible muscle contraction.

Generally, $2 \mathrm{~Hz}$ is chosen as a representative for low frequency, $100 \mathrm{~Hz}$ for high frequency, and $2 / 100 \mathrm{~Hz}$ for alternate low and high frequencies. According to the different frequencies of TEAS, patients were divided into three TEAS groups: TEAS- $2 \mathrm{~Hz}$ group, TEAS- $100 \mathrm{~Hz}$ group and TEAS-2/100 Hz group. In each group, the frequency of TEAS used before TVOR and ET was consistent. Patients in the control group followed the routine procedure of IVF treatment and no TEAS was applied. According to our pilot experiment, two treatments (before TVOR and ET) were sufficient to provide therapeutic benefits. Since patients could know whether they received treatment and detect the frequencies of TEAS, they were not blinded.

\section{Collection of the follicular fluids}

The long agonist protocol for controlled ovarian hyperstimulation $(\mathrm{COH})$ was used as previously described. ${ }^{23}$ Briefly, $\mathrm{COH}$ was performed by administration of recombinant follicle stimulating hormone (r-FSH, Gonal-F, Serono International S.A., Geneva, Switzerland) after pituitary suppression with triptorelin (Serono) started in the midluteal phase of the 


\section{ACCEPTED MANUSCRIPT}

preceding cycle. The dosages of gonadotrophins were individualized according to $E_{2}$ levels and trans-vaginally ultrasonic measurements of the follicles. When at least three follicles had reached the diameters of $16-18 \mathrm{~mm}$, ovulation was induced by administration of 10,000 IU human chorionic gonadotropin (HCG, Libao Biochemistry Co., Zhuhai, China). Trans-vaginal oocyte aspiration was performed with ultrasound guidance under general anesthesia $36 \mathrm{~h}$ after injection of HCG. The follicular fluids were sampled by trans-vaginal ultrasound-guided puncture and aspiration of the follicles with diameters of $16-18 \mathrm{~mm}$. Those follicular fluids samples that did not contain any visible blood contamination were used in this study. The follicular fluids samples were immediately centrifuged for $10 \mathrm{~min}$ at 550g. The supernatants were stored at $-80^{\circ} \mathrm{C}$ until analysis.

\section{Index and method}

The oocytes' developmental competence was evaluated by obtaining the numbers of metaphase II oocytes, normally fertilized zygotes and good quality embryos. The zygotes were checked 17-20 hours after fertilization, for two pronuclei (2PN) and polar bodies to assess fertilization. The good quality embryo was defined as: $7-9$ cells on Day $3,<10 \%$ fragmentation and equally sized mononucleated blastomeres. The clinical pregnancy rate (CPR), the primary outcome, was defined as the presence of at least one gestational sac or fetal heartbeat, confirmed by trans-vaginal ultrasound. Implantation rate (IR, defined as the number of gestational sacs per number of transferred oocytes) and live birth rate (LBR, defined as the presence of a baby born alive after 24 weeks gestation) for all the patients were also obtained. The levels of neuropeptide Y (NPY), transforming growth factor alpha (TGF-alpha) and granulocyte colony-stimulating factor (G-CSF) in the follicular fluids were 


\section{ACCEPTED MANUSCRIPT}

measured with enzyme-linked immunosorbent assay (ELISA, RayBio, GA, U.S.A).

\section{Sample size and data analysis}

The sample size was analyzed using Power Analysis and Sample Size (PASS 11.0). According to the records, a CPR of 0.38 was estimated in the control population and a CPR of $0.40,0.42$ and 0.57 was respectively estimated in TEAS- $2 \mathrm{~Hz}$ population, TEAS- $100 \mathrm{~Hz}$ population, and TEAS-2/100 Hz population. A sample size of 393 achieves $90 \%$ power to detect an effect size (W) of 0.19 using a 3 degrees of freedom Chi-Square Test with a significance level (alpha) of 0.05 .

Data were analyzed by an independent statistician using Statistical Package for Social Sciences (SPSS 19.0 for Windows). One-way analysis of variance (ANOVA) was used to evaluate statistical significances of continuous data. Chi-square test was used to compare categorical data. For all analyses, significance was set at $P<0.05$.

\section{Results}

\section{The sample population and baseline characteristics}

After the included 481 infertile women with bilateral tubal blockage were randomized, the control group had 120 cases, TEAS-2 Hz group 121 cases, TEAS-100 Hz group 119 cases and TEAS-2/100 Hz group 121 cases. The ET treatment was conducted three days after TVOR. In the statistical analysis, $11,13,8$ and 7 cases respectively in the control group, TEAS-2 Hz group, TEAS-100 Hz group and TEAS- $2 / 100 \mathrm{~Hz}$ group were excluded, as these patients did not complete ET. As shown in Table 1, there were no significant differences between the control group, TEAS-2 $\mathrm{Hz}$ group, TEAS- $100 \mathrm{~Hz}$ group and TEAS-2/100 $\mathrm{Hz}$ group on all the 
baseline characteristics $(P>0.05)$.

Table 1. The baseline characteristics of the participants

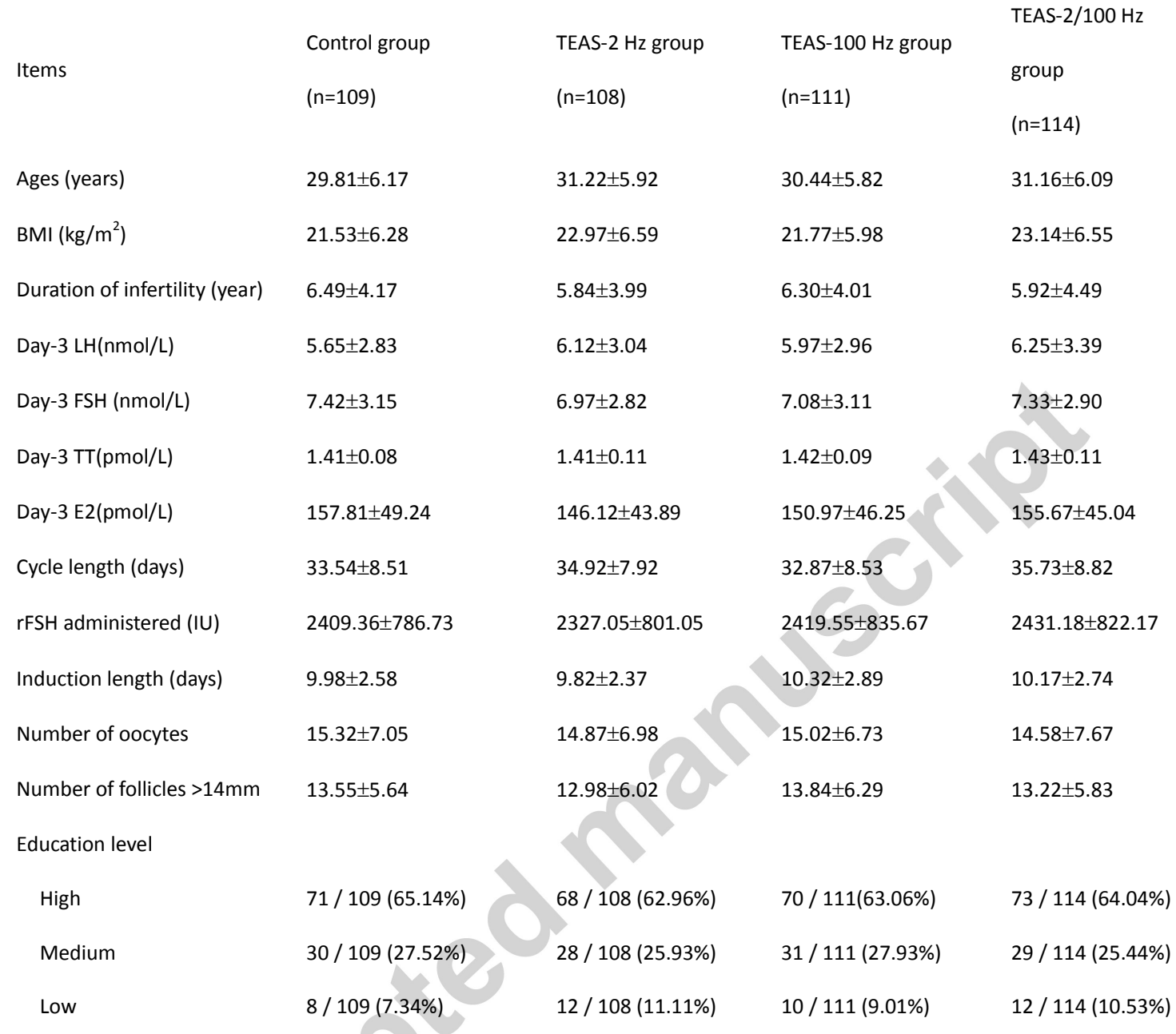

Note: Data were shown as mean \pm S.D. TEAS: transcutaneous electrical acupoint stimulation; BMI: body mass index; Day-3: the 3rd day of spontaneous menstrual cycle; LH: luteinizing hormone; FSH: follicle stimulating hormone; TT: total testosterone; E2: estradiol; r-FSH: recombinant FSH.

\section{The oocytes developmental competence}

As shown in Table 2, there were no significant differences between the control, TEAS-2 Hz, TEAS-100 Hz and TEAS-2/100 Hz groups on the numbers of metaphase II oocytes, normally fertilized zygotes or good quality embryos $(P>0.05)$. 


\section{ACCEPTED MANUSCRIPT}

Table 2: The oocytes developmental competence and the outcomes of in vitro fertilization.

\begin{tabular}{|c|c|c|c|c|}
\hline Items & $\begin{array}{l}\text { Control group } \\
(n=109)\end{array}$ & $\begin{array}{l}\text { TEAS- } 2 \mathrm{~Hz} \text { group } \\
(\mathrm{n}=108)\end{array}$ & $\begin{array}{l}\text { TEAS-100 Hz group } \\
(n=111)\end{array}$ & $\begin{array}{l}\text { TEAS-2/100 } \\
\text { group } \\
(n=114)\end{array}$ \\
\hline $\begin{array}{l}\text { Number of metaphase II } \\
\text { oocytes }\end{array}$ & $11.67 \pm 4.19$ & $11.85 \pm 4.78$ & $10.95 \pm 4.34$ & $11.21 \pm 4.59$ \\
\hline $\begin{array}{l}\text { Number of normally fertilized } \\
\text { zygotes }\end{array}$ & $7.62 \pm 5.73$ & $8.04 \pm 6.11$ & $7.90 \pm 5.88$ & $7.48 \pm 6.02$ \\
\hline $\begin{array}{l}\text { Number of good quality } \\
\text { embryos }\end{array}$ & $3.49 \pm 2.23$ & $3.31 \pm 2.19$ & $3.62 \pm 2.05$ & $3.54 \pm 1.99$ \\
\hline Clinical pregnancy rate (\%) & 46 / 109 (42.20\%) & 46 / 108 (42.59\%) & 47 / 111 (42.34\%) & $\begin{array}{l}64 / 114(56.14 \%) * \# \\
\$\end{array}$ \\
\hline Implantation rate (\%) & 54 / 217 (24.88\%) & 55 / 219 (25.11\%) & 53 / 223 (23.77\%) & $\begin{array}{l}82 / 231(35.50 \%) * \# \\
\$\end{array}$ \\
\hline Live birth rate (\%) & 38 / 109 (34.86\%) & 38 / 108 (35.19\%) & 39 / 111 (35.14\%) & $55 / 114(48.25 \%) * \#$ \\
\hline
\end{tabular}

Note: Data were shown as mean \pm S.D. ${ }^{*} P<0.05$, compared with the control group; ${ }^{\#} P<0.05$ compared with the TEAS- $2 \mathrm{~Hz}$ group; ${ }^{\$} P<0.05$ compared with the TEAS- $100 \mathrm{~Hz}$ group. TEAS: transcutaneous electrical acupoint stimulation.

\section{IVF outcomes}

As shown in Table 2, the CPR, IR and LBR of TEAS-2/100 Hz group were significantly higher than those of the other three groups respectively $(P<0.05)$. No significant differences existed between the control, TEAS-2 $\mathrm{Hz}$ and TEAS-100 $\mathrm{Hz}$ groups on the CPR, IR or LBR $(P>0.05)$ (Table 2).

\section{NPY, TGF-alpha and G-CSF levels in the follicular fluids}

As shown in Table 3, the NPY levels in the follicular fluids of the TEAS-2/100 Hz group were significantly higher than those of the other three groups respectively $(P<0.05)$. In the TEAS-100 Hz group, the NPY levels in the follicular fluids were significantly higher than those of the control and the TEAS- $2 \mathrm{~Hz}$ groups $(P<0.05)$ and no marked differences existed between the control and the TEAS- $2 \mathrm{~Hz}$ groups $(P>0.05)$. There were no significant 


\section{ACCEPTED MANUSCRIPT}

differences between all the groups on the TGF-alpha or G-CSF levels in the follicular fluids $(P>0.05)$.

Table 3: The levels of neuropeptide $\mathrm{Y}$, transforming growth factor alpha and granulocyte colony-stimulating factor in the follicular fluids.

$\begin{array}{lllll}\text { Items } & \begin{array}{l}\text { Control group } \\ (\mathrm{n}=109)\end{array} & \begin{array}{l}\text { TEAS-2 Hz group } \\ (\mathrm{n}=108)\end{array} & \begin{array}{l}\text { TEAS-100 Hz group } \\ (\mathrm{n}=111)\end{array} & \begin{array}{l}\text { TEAS-2/100 } \\ \text { group } \\ (\mathrm{n}=114)\end{array} \\ \mathrm{NPY}(\mathrm{ng} / \mathrm{ml}) & 23.70 \pm 7.95 & 25.66 \pm 8.19 & 34.07 \pm 9.10 * \# & 49.70 \pm 13.53 * \# \$ \\ \text { TGF-alpha }(\mathrm{pg} / \mathrm{ml}) & 29.13 \pm 6.38 & 30.61 \pm 7.23 & 30.04 \pm 6.76 & 29.93 \pm 6.42 \\ \text { G-CSF }(\mathrm{pg} / \mathrm{ml}) & 84.71 \pm 12.96 & 85.32 \pm 13.08 & 83.76 \pm 12.88 & 85.07 \pm 13.19\end{array}$

Note: Data were shown as mean \pm S.D. ${ }^{*} P<0.05$, compared with the control group; ${ }^{\#} P<0.05$ compared with the TEAS- $2 \mathrm{~Hz}$ group; ${ }^{\$} P<0.05$ compared with the TEAS-100 Hz group. TEAS: transcutaneous electrical acupoint stimulation; NPY: neuropeptide $Y ;$ TGF-alpha: transforming growth factor alpha; G-CSF: granulocyte colony-stimulating factor.

\section{Adverse events}

No side effects of the treatment were reported in any of the groups during the research and the follow-up period.

\section{Discussion}

In the present study, 481 infertile patients with bilateral tubal blockage referred for IVF were included to investigate whether TEAS can improve IVF outcomes. Patients were divided into four groups: TEAS- $2 \mathrm{~Hz}$ group, TEAS-100 Hz group, TEAS-2/100 Hz group and control group. We found the CPR, IR, LBR and NPY levels of the TEAS-2/100 Hz group were significantly higher than those of the other groups. However, there were no significant differences of the numbers of metaphase II oocytes, normally fertilized zygotes, early cleavage embryos and good quality embryos among the four groups.

TEAS is a new technique integrating traditional electro-acupuncture and new knowledge from advancing science. It can activate nerve endings and generate action potentials by 


\section{ACCEPTED MANUSCRIPT}

electrical stimulation. These signals, transmitted to spinal cord and brain, appear to stimulate the central nervous system to generate specific chemical substances to produce physiological effects. ${ }^{24,25}$ Effective frequency ranges from $2 \mathrm{~Hz}$ to $100 \mathrm{~Hz}$ for TEAS, and stimulation with different frequencies may generate totally different biological effects. ${ }^{26}$ Previous studies showed different frequencies of TEAS could generate totally different biological effects: low-frequency $(2 \mathrm{~Hz})$ electrical stimulation promoted the release of enkephalins, endorphins and endomorphin in the brain to interact with the $\mu$ - and $\delta$-opioid receptors; high-frequency $(100 \mathrm{~Hz})$ promoted the release of dynorphin in spinal cord to interact with the $\mathrm{k}$ opioid receptors; and alternate low and high frequencies $(2 / 100 \mathrm{~Hz})$ promoted the release of all four kinds of endogenous opioid peptides. ${ }^{27}$ TEAS treatment was found to improve CPR in the patients with decreased ovarian reserve during IVF. ${ }^{28}$ In the present study, we found TEAS using a frequency of $2 / 100 \mathrm{~Hz}$ significantly improved the CPR, IR and LBR of the IVF women.

The impact of acupuncture and electro-acupuncture on the clinical outcomes of IVF has been well studied. Positive effects have been shown in modulating the ovarian blood flow and the functions of the hypothalamus-pituitary-ovary system. ${ }^{29-31}$ One randomized, prospective, controlled clinical study found that clinical pregnancy rate and ongoing pregnancy rate more than doubled by luteal-phase acupuncture. ${ }^{32}$ Another study showed acupuncture on the day of ET increased clinical pregnancy and ongoing pregnancy rates by about $50 \%{ }^{11}$ However, because the acupoints, acupuncturists, patients and treatment time in these studies were varied, it was difficult to compare acupuncture with TEAS.

For patients who are unwilling to receive invasive or painful acupuncture treatment, TEAS is 


\section{ACCEPTED MANUSCRIPT}

potentially a good choice. In the TEAS treatment, the skin electrodes are placed on the patients' acupoints, instead of piercing the skin with the traditional acupuncture needles. TEAS can also increase the reproducibility of the acupuncture-like stimulation..$^{24,25}$ Compared with acupuncture, TEAS is easier to use: patients can even potentially use TEAS on their own under the guidance of acupuncturists. We did not find any adverse events in any group during the research and the follow-up period. This indicates TEAS is a safe intervention to improve the outcomes of IVF.

In the study, the levels of NPY, TGF-alpha and G-CSF in the follicular fluids of the IVF patients were measured to explore the potential mechanism underlying TEAS improving the outcomes of IVF. NPY may represent a link between nutrition and reproduction at the level of the central nervous system, ${ }^{33}$ and may serve as one of the molecular signlas integrating the control of metabolism and reproduction. ${ }^{34} \mathrm{NPY}$ may play a role in positive regulation of gonadotropin-releasing hormone $(\mathrm{GnRH})$ throughout the neural axis and also up-regulate the LH cells in the pituitary. ${ }^{35} \mathrm{NPY}$ is also a key factor during the follicular growth, ovulation and regulating the functions of the hypothalamic-pituitary-ovarian axis. ${ }^{36-41}$ Electro-acupuncture was found to significantly improve the levels of NPY in the follicular fluids of the patients. ${ }^{42}$ The result is consistent with the present study.

TGF-alpha plays an important role in regulating the growth of follicles and the maturation of oocyte. ${ }^{43-45} \mathrm{G}-\mathrm{CSF}$, also known as colony-stimulating factor 3 (CSF3), is a biomarker of human oocyte developmental competence for embryo implantation. ${ }^{46-49}$ It has a higher discriminatory power to predict the ongoing pregnancy in multivariate logistic regression analysis for G-CSF levels in the follicular fluids, compared with embryo 


\section{ACCEPTED MANUSCRIPT}

morphology. ${ }^{50}$ It was found that the quality of porcine oocyte and embryonic viability were significantly improved when adding the human recombination G-CSF into porcine in vitro maturation medium. ${ }^{51}$ In the present study, the NPY levels in the follicular fluids of the TEAS-2/100 $\mathrm{Hz}$ group were significantly higher than those of the other groups, and there were no significant differences between all the four groups on the TGF-alpha or G-CSF levels in the follicular fluids. This could indicate that NPY, as opposed to one of the othese other molecules examined, may be the primary mediator of the improvement in outcomes of the IVF patients.

Although it has been reported that TEAS may improve IVF outcomes, the underlying mechanisms are not clear. Several mechanisms have been suggested to explain the role of acupuncture in IVF, such as increasing blood flow and ovarian steroid hormone secretion, reducing anxiety of patients, etc. In the present study, we investigated whether TEAS may improve IVF outcomes by influencing oocyte developmental competence, which is defined as the ability of oocyte to complete meiosis and undergo fertilization, embryo genesis and term development. We did not find any differences between the control, TEAS-2 Hz, TEAS-100 Hz and TEAS-2/100 Hz groups on the numbers of metaphase II oocytes, normally fertilized zygotes, early cleavage embryos or good quality embryos. Moreover, follicular fluid levels of TGF-alpha (reflecting oocyte maturation) and G-CSF (a biomarker of human oocyte developmental competence) showed no significant differences between all the four groups, indicating that TEAS influences IVF outcomes through mechanisms other than improving oocyte quality.

In conclusion, TEAS using a frequency of $2 / 100 \mathrm{~Hz}$ could help to improve the IVF outcomes 


\section{ACCEPTED MANUSCRIPT}

partly by increasing the NPY levels in the follicular fluids. These findings provide preliminary evidence to suggest that TEAS may be a new option for clinicians and infertile women during IVF treatment. Limitations of this study include lack of a placebo group, small sample size, short research period and the fact that patients included were only those with bilateral tubal blockage. A larger randomized, multicenter, double-blinded and placebo-controlled trial should be conducted in the near future to further confirm the effects of TEAS on the IVF outcomes and to further explore the potential underlying mechanism of this effect.

\section{Funding}

This work was supported by the Zhejiang Provincial Natural Science Foundation of China [Grant No. LR16H040001]; the Natural Science Foundation [Grant No. 81370761] and the special research fund for the public health welfare industry of health of China [No. 201302013].

\section{Conflicts of interest}

None.

\section{Reference}

1 Andersen AN, Goossens V, Gianaroli L, et al. Assisted reproductive technology in Europe, 2003. Results generated from European registers by ESHRE. Hum Reprod. 2007;22(6):1513-1525.

2 Harper J, Magli MC, Lundin K, Barratt CL, Brison D. When and how should new technology be introduced into the IVF laboratory? Hum Reprod. 2012;27(2):303-313.

3 Manheimer E, van der Windt D, Cheng K, et al. The effects of acupuncture on rates of clinical pregnancy among women undergoing in vitro fertilization: a systematic review and meta-analysis. Hum Reprod Update. 2013;19(6):696-713.

4 Zhang Y, Fu Y, Han F, et al. The effect of complementary and alternative medicine on 
subfertile women with in vitro fertilization. Evid Based Complement Alternat Med. 2014; 2014(1):68-78.

Shen C, Wu M, Shu D, Zhao X, Gao Y. The Role of Acupuncture in in vitro Fertilization: A Systematic Review and Meta-Analysis. Gynecol Obstet Invest. 2015;79(1):1-12.

Weeks K, Gaspard G. The use of acupuncture with in vitro fertilization as a treatment for female subfertility. J Altern Complement Med. 2013;19(8):732.

Hullender Rubin LE, Opsahl MS, Taylor-Swanson L, Ackerman DL. Acupuncture and in vitro fertilization: a retrospective chart review. J Altern Complement Med. 2013;19(7):637-643. Qu F, Zhou J, Ren RX. Effects of acupuncture on the outcomes of in vitro fertilization: a systematic review and meta-analysis. J Altern Complement Med. 2012;18(5):429-439. Hopton AK, Curnoe S, Kanaan M, Macpherson H. Acupuncture in practice: mapping the providers, the patients and the settings in a national cross-sectional survey. BMJ Open. 2012;2(1):e000456.

Stener-Victorin E, Humaidan P. Use of acupuncture in female infertility and a summary of recent acupuncture studies related to embryo transfer. Acupunct Med. 2006;24(4):157-163.

Westergaard LG, Mao Q, Krogslund M, et al. Acupuncture on the day of embryo transfer significantly improves the reproductive outcome in infertile women: a prospective, randomized trial. Fertil Steril. 2006;85(5):1341-1346.

Ernst G, Strzyz H, Hagmeister H. Incidence of adverse effects during acupuncture therapy-a multicentre survey. Complement Ther Med. 2003;11(2):93-97.

Witt CM, Pach D, Brinkhaus B, et al. Safety of acupuncture: results of a prospective observational study with 229,230 patients and introduction of a medical information and consent form. Forsch Komplementmed. 2009;16(2):91-97.

Leibing E, Leonhardt U, Köster G, et al. Acupuncture treatment of chronic low-back pain-a randomized, blinded, placebo-controlled trial with 9-month follow-up. Pain. 2002;96(1):189-196.

Penetar DM, Burgos-Robles A, Trksak GH, et al. Effects of transcutaneous electric acupoint stimulation on drug use and responses to cue-induced craving: a pilot study. Chin Med. 2012;7(1):1.

Chao AS, Chao A, Wang TH, et al. Pain relief by applying transcutaneous electrical nerve stimulation (TENS) on acupuncture points during the first stage of labor: a randomized double-blind placebo-controlled trial. Pain. 2007;127(3):214-220.

Ma D, Han JS, Diao QH, et al. Transcutaneous electrical acupoint stimulation for the treatment of withdrawal syndrome in heroin addicts. Pain Med. 2015;16(5):839-848.

Omura Y, Chen Y, Lermand O, et al. Effects of transcutaneous electrical stimulation (1 pulse/sec) through custom-made disposable surface electrodes covering Omura's ST36 area of both legs on normal cell telomeres, oncogen C-fosAb2, integrin alpha5beta1, chlamydia trachomatis, etc. in breast cancer \& alzheimer patients. Acupunct Electrother Res. 2010;35(3-4):147-185.

Tsay SL, Cho YC, Chen MI. Acupressure and transcutaneous electrical acupoint stimulation in improving fatigue, sleep quality and depression in hemodialysis patients. Am J Chin Med. 2004;32(03):407-416.

Moher D, Schulz KF, Altman DG. The CONSORT statement: revised recommendations for improving the quality of reports of parallel-group randomised trials. Lancet. 
2001;357(9263):1191-1194.

Bian ZX, Chang YH. Revised STRICTA as an extension of the CONSORT statement: more items should be involved in the checklist. J Altern Complement Med. 2011;17(2):97-98. MacPherson H, Altman DG, Hammerschlag R, et al. Revised STandards for Reporting Interventions in Clinical Trials of Acupuncture (STRICTA): extending the CONSORT statement. PLoS Med. 2010;7(6):e1000261.

Qu F, Wang FF, Lu XE, et al. Altered aquaporin expression in women with polycystic ovary syndrome: hyperandrogenism in follicular fluid inhibits aquaporin-9 in granulosa cells through the phosphatidylinositol 3-kinase pathway. Hum Reprod. 2010;25(6):1441-1450. Zhang R, Feng XJ, Guan Q, et al. Increase of success rate for women undergoing embryo transfer by transcutaneous electrical acupoint stimulation: a prospective randomized placebo-controlled study. Fertil Steril. 2011;96(4):912-916.

Zhang Q, Gao Z, Wang H, et al. The effect of pre-treatment with transcutaneous electrical acupoint stimulation on the quality of recovery after ambulatory breast surgery: a prospective, randomised controlled trial. Anaesthesia. 2014;69(8):832-839. Han J, Chen X, Sun S, et al. Effect of low-and high-frequency TENS on Met-enkephalin-Arg-Phe and dynorphin A immunoreactivity in human lumbar CSF. Pain. 1991;47(3):295-298. Han JS. Acupuncture: neuropeptide release produced by electrical stimulation of different frequencies. Trends Neurosci. 2003;26(1):17-22.

Zheng $\mathrm{Y}$, Feng $\mathrm{X}, \mathrm{Mi} \mathrm{H}$, et al. Effects of transcutaneous electrical acupoint stimulation on ovarian reserve of patients with diminished ovarian reserve in in vitro fertilization and embryo transfer cycles. J Obstet Gynaecol Res. 2015;41(12):1905-1911.

STENER-VICTORIN E, WALDENSTRÖM U, Tägnfors $U$, et al. Effects of electro-acupuncture on anovulation in women with polycystic ovary syndrome. Acta Obstet Gynecol Scand. 2000;79(3):180-188.

Stener-Victorin E, Kobayashi R, Watanabe O, Lundeberg T, Kurosawa M. Effect of electro-acupuncture stimulation of different frequencies and intensities on ovarian blood flow in anaesthetized rats with steroid-induced polycystic ovaries. Reprod Biol Endocrinol. 2004;2(1):1.

Stener-Victorin E, Kobayashi R, Kurosawa M. Ovarian blood flow responses to electro-acupuncture stimulation at different frequencies and intensities in anaesthetized rats. Auton Neurosci. 2003;108(1):50-56. Dieterle S, Ying G, Hatzmann W, Neuer A. Effect of acupuncture on the outcome of in vitro fertilization and intracytoplasmic sperm injection: a randomized, prospective, controlled clinical study. Fertil Steril. 2006;85(5):1347-1351. Wojcik-Gladysz A, Polkowska J. Neuropeptide Y--a neuromodulatory link between nutrition and reproduction at the central nervous system level. Reprod Biol. 2006;6 Suppl 2(21-28. Crown A, Clifton DK, Steiner RA. Neuropeptide signaling in the integration of metabolism and reproduction. Neuroendocrinology. 2007;86(3):175-182.

Subhedar N, Gaikwad A, Biju KC, Saha S. Role of neuropeptide Y (NPY) in the regulation of reproduction: study based on catfish model. Fish Physiol Biochem. 2005;31(2-3):167-172. McNeill DL, Burden HW. Peripheral pathways for neuropeptide $Y$ - and cholecystokinin-8-immunoreactive nerves innervating the rat ovary. Neurosci Lett. 
1987;80(1):27-32.

Jorgensen JC, Sheikh SP, Forman A, et al. Neuropeptide $Y$ in the human female genital tract: localization and biological action. Am J Physiol. 1989;257(2 Pt 1):E220-227. Jorgensen JC, O'Hare MM, Andersen CY. Demonstration of neuropeptide $\mathrm{Y}$ and its precursor in plasma and follicular fluid. Endocrinology. 1990;127(4):1682-1688. Tatemoto K, Carlquist M, Mutt V. Neuropeptide Y--a novel brain peptide with structural similarities to peptide YY and pancreatic polypeptide. Nature. 1982;296(5858):659-660. Markiewicz W, Jaroszewski JJ, Bossowska A, Majewski M. NPY: its occurrence and relevance in the female reproductive system. Folia Histochem Cytobiol. 2003;41(4):183-192. Barreca A, Valli B, Cesarone A, et al. Effects of the neuropeptide $Y$ on estradiol and progesterone secretion by human granulosa cells in culture. Fertil Steril. 1998;70(2):320-325. Stener-Victorin E, Waldenstrom $\mathrm{U}$, Wikland $\mathrm{M}$, et al. Electro-acupuncture as a peroperative analgesic method and its effects on implantation rate and neuropeptide $Y$ concentrations in follicular fluid. Hum Reprod. 2003;18(7):1454-1460.

Tamura M, Sasano H, Suzuki T, et al. Expression of epidermal growth factors and epidermal growth factor receptor in normal cycling human ovaries. Hum Reprod. 1995;10(7):1891-1896.

Qu J, Nisolle M, Donnez J. Expression of transforming growth factor-alpha, epidermal growth factor, and epidermal growth factor receptor in follicles of human ovarian tissue before and after cryopreservation. Fertil Steril. 2000;74(1):113-121.

Reeka N, Berg FD, Brucker C. Presence of transforming growth factor alpha and epidermal growth factor in human ovarian tissue and follicular fluid. Hum Reprod.

1998;13(8):2199-2205.

Ledee N, Petitbarat M, Rahmati M, et al. New pre-conception immune biomarkers for clinical practice: interleukin-18, interleukin-15 and TWEAK on the endometrial side, G-CSF on the follicular side. J Reprod Immunol. 2011;88(2):118-123.

47 Ledee N, Munaut C, Serazin V, et al. Performance evaluation of microbead and ELISA assays for follicular G-CSF: a non-invasive biomarker of oocyte developmental competence for embryo implantation. J Reprod Immunol. 2010;86(2):126-132.

Ledee N, Frydman R, Osipova A, et al. Levels of follicular G-CSF and interleukin-15 appear as noninvasive biomarkers of subsequent successful birth in modified natural in vitro fertilization/intracytoplasmic sperm injection cycles. Fertil Steril. 2011;95(1):94-98. Salmassi A, Schmutzler AG, Schaefer S, et al. Is granulocyte colony-stimulating factor level predictive for human IVF outcome? Hum Reprod. 2005;20(9):2434-2440. Ledee N, Gridelet V, Ravet S, et al. Impact of follicular G-CSF quantification on subsequent embryo transfer decisions: a proof of concept study. Hum Reprod. 2013;28(2):406-413. Cai L, Lee E, Hyun SH. 303 human recombination granulocyte-colony stimulating factor (hrg-csf) have beneficial effects on porcine oocytes quality during in vitro maturation and subsequent viability of embryonic development. Reprod Fertil Dev. 2014;27(1):240. 